\title{
LAND-USE DYNAMICS ANALYSIS BASED ON SPATIAL INTERACTIONS MODELS AND SATELLITE IMAGES TIME-SERIES
}

\author{
J.-F. Girres ${ }^{1}$ \\ ${ }^{1}$ Université Paul-Valéry Montpellier 3, IRD, UMR GRED 220, Route de Mende, 34199 Montpellier, France \\ jean-francois.girres@univ-montp3.fr
}

\section{Commission IV, WG IV/1}

KEY WORDS: Spatial Interactions, Land-use, Remote Sensing, Retail Gravitation

\begin{abstract}
:
This article presents a research agenda in the domain of the analysis of land-use dynamics. The proposed research intends to develop spatial interactions models in order to understand and simulate land-use dynamics that operate on particular territories. The proposed approach takes into account the main principles of spatial interactions models, such as Reilly's theory of retail gravitation, combined with land-use coverages extracted from satellite images time-series. The study of the relationship between land-use dynamics with forces of attraction estimated from spatial interactions models can help to better understand how land-use changes are distributed. The proposed approach can also be used to simulate potential evolutions of land-use around time. Finally, this article exposes the contextual limits of the proposed approach, and also presents associated opportunities of research and study areas for application.
\end{abstract}

\section{INTRODUCTION}

Recent advances in the field of satellite image processing, with in particular the appearance of deep learning techniques, based on convolutional neural networks (Zhang et al., 2016), have profoundly changed the extraction potential of information in satellite images. This technological development, coupled with an increasingly diversified range of satellite images (particularly in terms of spatial or temporal resolutions) opens up new perspectives for the representation of land-use and the analysis of its dynamics.

This context offers in particular the possibility of more precisely discriminating land-use patches at different scales of representation, and thus better perceiving the changes that accompany land-use over time. For example, the use of Sentinel 2 images time-series, coupled with automated processing chains, allows regular revisiting of land-use throughout France (Inglada et al., 2017) with a spatial resolution of 10 meters, as exposed on the area of Montpellier (France) in 2017 (figure 1).

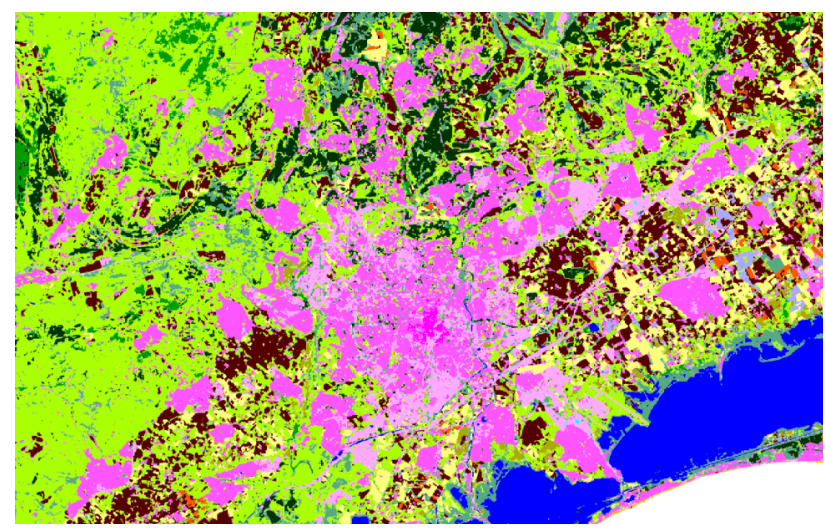

Figure 1. Land-use cartography on the area of Montpellier in 2017, extracted from Sentinel-2 satellite images (source: CES OSO).
These recent advances therefore contribute to an improved understanding of the territories and their dynamics. However, to better perceive the changes taking place in a territory, it seems necessary to go further.

Indeed, to understand the dynamics of land-use that operate on a territory, it looks important not to limit spatial analysis to a static description of land-use, even in a diachronic manner. Issues related to the analysis of land-use dynamics can be investigated through the angle of spatial interactions (Wilson, 1998) and especially by the use of gravity models (Reilly, 1931; Huff, 1964). These models are directly inspired by Newton's universal law of gravitation, where the gravity is deployed around cores, which are generally urban centers.

For instance, figure 2 illustrates land-use changes (in red) observed around the city of Montpellier (France) between 2006 and 2012 in the Corine Land-Cover database, at different distances of the town center $(2,4,6$, and $8 \mathrm{~km})$.

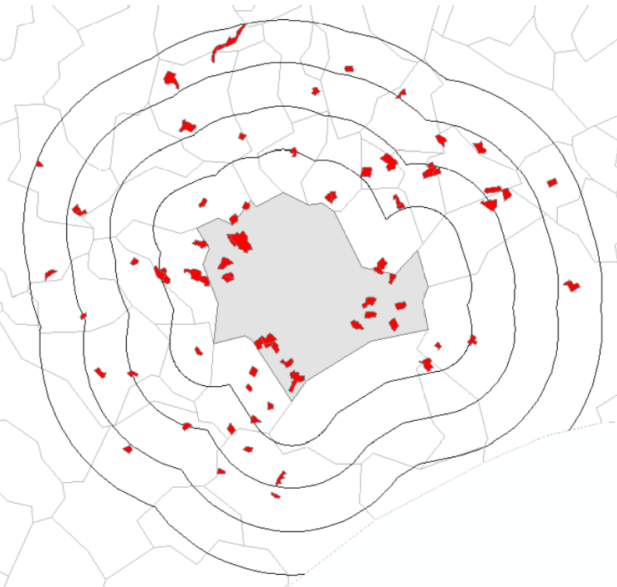

Figure 2. Land-use changes observed between 2006 and 2012 around the city of Montpellier (France) in the Corine Land Cover database 
Results exposed in table 1 show that the surface of land-use changes decreases when the distance to the town center increase. For instance, the total surface of land-use changes is about $2.8 \mathrm{sq}$. $\mathrm{km}$. within the city of Montpellier. This surface deceases at $2.1 \mathrm{sq} . \mathrm{km}$. within a distance of $2 \mathrm{~km}$, and at $1.6 \mathrm{sq}$. $\mathrm{km}$. between 4 and $6 \mathrm{~km}$.

\begin{tabular}{|c|c|}
\hline $\begin{array}{c}\text { Distance around } \\
\text { Montpellier }\end{array}$ & $\begin{array}{c}\text { Surface of Land-Use } \\
\text { Changes }\end{array}$ \\
\hline$<0 \mathrm{~km}$ & 2.84 sq. km. \\
$0-2 \mathrm{~km}$ & 2.06 sq. km. \\
$2-4 \mathrm{~km}$ & 1.96 sq. km. \\
$4-6 \mathrm{~km}$ & 1.60 sq. km. \\
$6-8 \mathrm{~km}$ & 1.44 sq. km. \\
\hline
\end{tabular}

Table 1. Surface of land-use changes between 2006 and 2012 according to the distance around the city of Montpellier (France)

These first results clearly show that the intensity of land-use changes can be explained by the distance to gravitation cores, such as urban centers. Such observations also make it possible to forecast land-use dynamics, using simulation techniques. These issues reach the domain of Land-Use Transport Interaction (LUTI) models, which have been largely developed in the past decades, with numerous contributions based on the theory of retail gravitation (Wegener and Fuerst, 2004).

Thus, this research intends to propose approaches to understand and explain land-uses changes based on spatial interactions models, in order to forecast land-use dynamics. The originality of this research is that it intends to benefit from land-use mapping extracted from satellite images time-series, with high spatial and temporal resolutions.

After presenting the approach used to understand the distribution of land-use dynamics using spatial interaction models, a methodology is proposed, followed by a research agenda, before concluding.

\section{APPROACH}

To understand the dynamics of land-use that operate on a territory, it is necessary to relate these dynamics with the spatial interactions that appear between different land-use units, which structure its organization and evolution. For instance, it is well known, notably through the theory of retail gravitation, introduced by Reilly (1931), that urban centers deploy capacities of attraction and emission on the peripheries which surround them. These attraction and emission capacities are manifested in particular by the circulation of material from/to the urban center (e.g. population flows, telephone calls, letters), as well as by specific changes in terms of land-use (e.g. artificialization of soils in the peripheries).

Understanding the dynamics of land-use, beyond a purely descriptive analysis, therefore implies understanding the spatial interactions that operate on a territory, and modeling the forces that polarize them. Traditionally, the analysis of spatial interactions mainly focuses on the relationships between urban centers and their peripheries. To estimate the interactions which take place between several urban centers, several gravity models can be used.
The model introduced by Reilly (1931) defines the main principles of spatial interactions approaches based on gravitation theory.

Reilly's model involves to estimate several parameters:

- the weight of each core (which can be estimated by the population of a urban center for example),

- the distance which separates each core from the other neighboring places.

Finally, Reilly's model can be presented using equation 1 .

$$
F_{i j}=M_{j} / d_{i j}{ }^{2}
$$

where

$F_{i j}$ is the attraction force of the center $j$ on the place $\mathrm{i}$

$\mathrm{M}_{\mathrm{j}}$ is the weight of the center $\mathrm{j}$

$\mathrm{d}_{\mathrm{ij}}$ is the distance between $\mathrm{i}$ and $\mathrm{j}$

Generally, the weight of each urban center is determined using its population, but it can also be inferred through an extraction of the surface of built-up areas (and eventually its components). This approach has already been proposed in numerous contributions (Mossoux et al., 2018).

The distance between the different centers can be determined using the Euclidean distance, or other metrics (e.g. Manhattan distance). Traditionally, the square of the distance is used in Reilly's gravitation model to determine the attraction force of a center on a neighboring position.

By calibrating a model from these information, it may be possible to estimate the spatial interactions operating between different urban centers, and thus quantify specific flows (e.g. workflows), or determining the area of influence of urban centers on their surrounding peripheries, using a field of gravitation such as the one exposed in figure 3 .

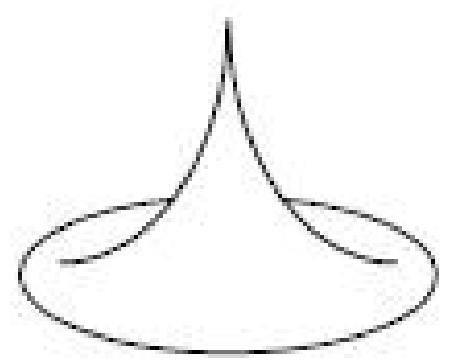

Figure 3. Principle of modeling of the area of influence of a core on its periphery, using Reilly's model.

Several extensions of Reilly's theory of retail gravitation have been proposed to model spatial interactions, such as the models proposed by Stewart (1942), Huff (1964), or Wilson (1967).

One of the most famous gravity model was proposed by Huff (1964), who introduced the concept of concurrency in the modeling of spatial interactions. He proposed to model the area of influence of urban centers using fuzzy limits estimated by probabilities of attraction of a center on neighboring places. 
To estimate the probability of attraction of a center on surrounding positions, Huff (1964) proposes to define the Opportunity offered by each center, using a generalized Reilly's model, as exposed in equation 2.

$$
\mathrm{O}_{\mathrm{ij}}=\mathrm{M}_{\mathrm{j}} / \mathrm{d}_{\mathrm{ij}}{ }^{\alpha}
$$

where $\quad \mathrm{O}_{\mathrm{ij}}$ is the opportunity offered by the center $\mathrm{j}$ on the place $\mathrm{i}$

$\mathrm{M}_{\mathrm{j}}$ is the weight of the center $\mathrm{j}$

$\mathrm{d}_{\mathrm{ij}}$ is the distance between $\mathrm{i}$ and $\mathrm{j}$

The Total Potential of relationships is defined by the sum of all Opportunities, as exposed in equation 2.

$$
\mathrm{T}_{\mathrm{i}}=\Sigma_{\mathrm{k}} \mathrm{O}_{\mathrm{ik}}
$$

where $\quad T_{i}$ is the total potential of opportunities from the place $\mathrm{i}$

$\mathrm{k}$ is the number of centers

Finally, the Probability of attraction of a center on surrounding positions is defined by equation 4 .

$$
P_{i j}=O_{i j} / T_{i}
$$

where

$P_{i j}$ is the probability of attraction offered by the center $\mathrm{j}$ on the place $\mathrm{i}$

$\mathrm{O}_{\mathrm{ij}}$ is the opportunity offered by the center $\mathrm{j}$ on the place $\mathrm{i}$

$T_{i}$ is the total potential of opportunities from the place $\mathrm{i}$

The model proposed by Huff (1964) allows to estimate areas of influence determined by probabilistic fields of gravitation. For instance, the theoretical areas of influence of the three main cities of the department of Hérault (France) can be determined using their population, as exposed in figure 4.

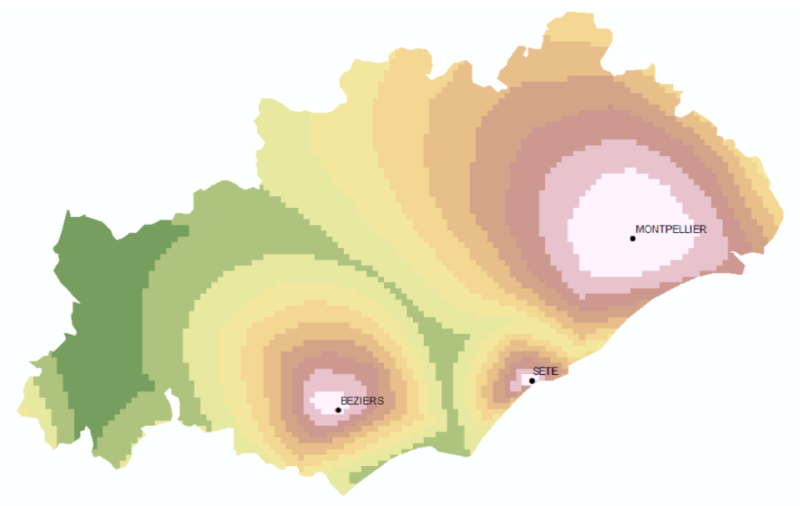

Figure 4. Probabilistic area of influence of the three main cities of the department of Hérault (France), using Huff model.
Based on the relative position of land-use patches in relation to gravitational core, such as urban centers, it may be possible to analyze more precisely how land-use dynamics operate on particular territories.

In this context, the exploitation of land-use time series also makes it possible to understand how spatial interactions manifest themselves in terms of evolution of land-use classes, according to different parameters (distance to urban center, weight of the surrounding urban centers). So, this research intends to find relationships between spatial interaction models and evolutions of land-use classes over time.

However, a contextual dimension must also be considered to quantify the interactions around urban centers. Indeed, depending on its local context, the surface of built-up areas will not allow to determine its weight precisely (particularly through its population) and the effect of the distance will also vary in terms of of land-use dynamics.

For example, the built-up area of a North American city like Atlanta is twenty times bigger that the one of Barcelona, with a comparable population (around 2.7 million inhabitants), as exposed by Bertaud and Richardson (2004) in figure 5.

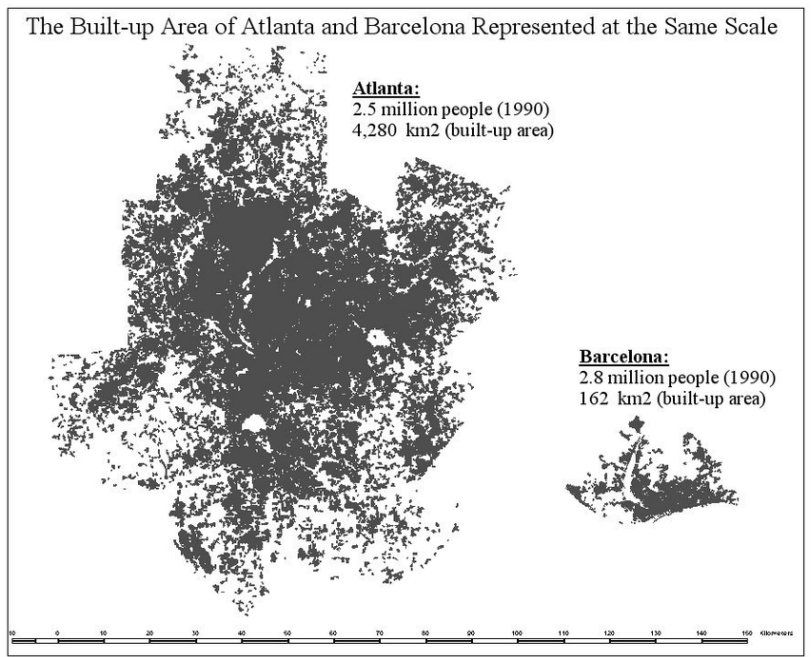

Figure 5. Comparison of the built-up areas of Atlanta and Barcelona, from Bertaud and Richardson (2004)

This example demonstrates that the specific context of urban areas must be taken into account to calibrate a spatial interactions model in an appropriate and realistic way.

\section{METHODOLOGY}

In order to study the relationship between land-use dynamics and spatial interaction models, a methodology is described in this section. The proposed methodology has not been yet implemented. So, it is supposed to be consolidated in the future.

This proposed methodology is structured in five steps, as presented in Figure 6. 


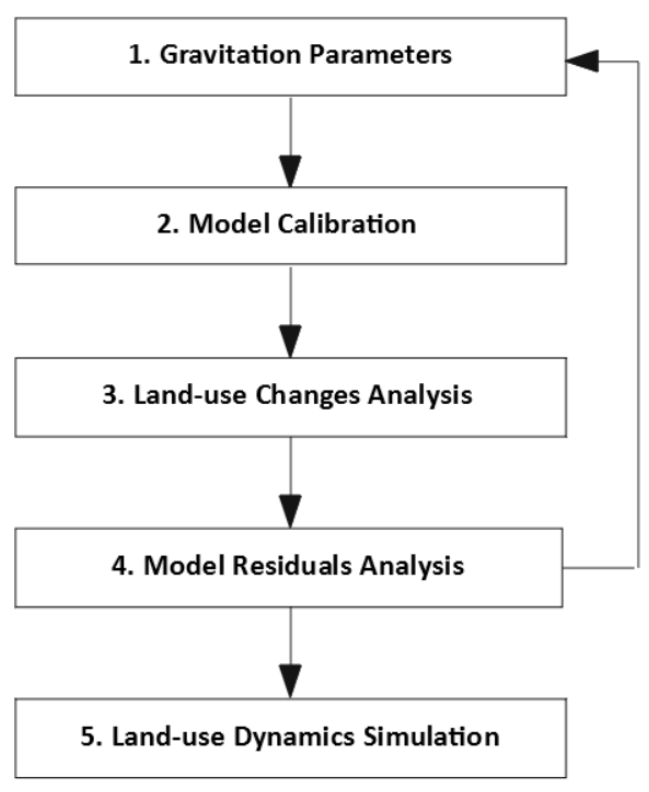

Figure 5. Proposed methodology to analyze land-use dynamics using spatial interaction models

\subsection{Definition of the gravitation parameters}

Firstly, it is necessary to identify the factors which can be considered as structuring forces on a given territory. These factors (or driving forces) can be considered as the parameters of the spatial interaction model, as they are supposed to generate emission and reception capacities. Naturally, urban areas will be used as the main cores around which the principles of gravitation will be built.

To initialize the weight of these centers, different indicators can be used, and even crossed. The first indicator is the surface of the urban area. But this indicator can also be supplemented with socio-demographic variables (if available), which can characterize the attractiveness of urban centers. For example, the population density, or the number of jobs can be used to define the attractiveness of urban areas.

Beyond the study of the attractiveness of urban centers, the study of the proximity to certain strategic resources could also be implemented. For example, the distance to the coastline could also be included in the model, if we consider the attractiveness that coastal zones can provide, and the impact that this can have in terms of artificialization of soils.

Once the parameters structuring the principles of gravitation defined, the calibration of the spatial interactions model can be realized.

\subsection{Calibration of the spatial interactions model}

As mentioned above, the gravitation parameters used by the spatial interactions model can vary according to the specific characteristics of the study area. As a consequence, it will therefore be necessary to test different settings of the spatial interactions model, in order to define the most appropriate calibration to analyze land-use dynamics.
This study will be based on a sensitivity analysis of the two main parameters of the gravity model, namely the weight of the cores and the distance used. Different sets of weights and distances will be tested and compared with the quantity of landuse evolution patches extracted from time-series of satellite images (such as the OSO product from CESBIO for example).

Based on this multi-dimensional sensitivity analysis, we will seek to calibrate the spatial interactions model with the appropriate parameters to analyze land-use dynamics around.

\subsection{Analysis of land use dynamics}

Once the spatial interaction model has been calibrated, the analysis of land use dynamics can be carried out. These analyzes will seek to establish the relationship between (1) changes in land-use (from class A to class B) and (2) distance to one or more gravitation cores (as previously established).

Depending on the areas of investigation, the land-use classes studied may vary, particularly depending on local issues. For example, in the Hérault department, the question of the artificialization of soils (by the transformation of agricultural areas into urbanized areas) or that of agricultural abandonment (by the transformation of agricultural land into natural areas) will be more investigated. On the other hand, in other areas, such as in the Amazonian area, the question of deforestation will be particularly studied.

\subsection{Analysis of the residuals of the model}

In addition to the analysis of land-use dynamics presented above, a particular attention will be provided on the study of the residuals of the spatial interactions model. Indeed, the detection of a too important amount of land-use changes in areas not predicted by the model could reveal gaps in terms of initial assumptions (through the definition of gravitational cores) or calibration of the model. The analysis of the residuals could therefore make it possible to validate the spatial interactions model (if the residuals are acceptable) or else to modify it (by changing the initial assumptions or the calibration of the model) in order to reach a model more faithful to land-use changes.

\subsection{Simulation of land-use dynamics}

The last step of this methodology is intended to be forwardlooking, through the possibility of simulating future changes in terms of land-use, using consolidated spatial interactions model. The construction of prospective scenarios for land-use development will use spatial simulation tools, such as cellular automata or multi-agent systems, which have already been widely used in order to forecast land-use dynamics (Houet and Hubert-Moy, 2006; Li and Yeh, 2002).

\section{RESEARCH AGENDA}

As exposed above, the present research intends to study the relationships between spatial interaction models and land-use dynamics, extracted from time-series of satellite images. This research initially intends to model the spatial interactions operated from urban centers. However, it could be extended to other types of land-use classes with particular capacities of attraction and emission, such as coastlines for example. 
This work reaches several fields of research, such as Land-Use Transport Interactions (LUTI) models, or also methodological approaches that intend to extract indirect information from satellite images, as for example the works developed for predicting socio-economic variables (Jean et al., 2016; Georganos et al., 2019). This exploratory research aims in particular to enrich the knowledge automatically extracted from series of satellite images. The field of application of this work mainly focuses on the observation of regions with active landuse dynamics, but little information on the interactions between urban centers. More particularly, this research is intended to be developed in territories affected by important land-use changes, such as in Western Africa or in Amazonia.

Beyond the modeling of spatial interactions operated on a territory, and its manifestation in terms of land-use dynamics, this research also intends to propose methods of cartographic representation of spatial interactions, in particular through the representation of flows in maps.

Thus, this exploratory work aims to decline several lines of research: (1) modeling of spatial interactions around urban centers, (2) calibration of models within different geographical contexts, (3) application to other land-use classes, and (4) cartographic representation of flows at different scales.

\section{CONCLUSION}

To sum up, this research intends to develop spatial interactions models, based on gravitation theory, in order to analyze and forecast land-use dynamics extracted from satellite images timeseries. This research seeks to meet different disciplines, such as remote sensing, spatial analysis, or even cartography, with the perspective of a better understanding of land-use evolution on territories characterized by important dynamics.

\section{REFERENCES}

Bertaud, A., Richardson, H., W., 2004. Transit and Density: Atlanta, the United States and Western Europe. In H., W. Richardson, C., H. C., Bae (eds): Urban Sprawl in Western Europe and the United Sates, Urban Planning and Environment, Ashgate, $325 \mathrm{p}$.

Georganos, S., Gadiaga, A. N., Linard, C., Grippa, T., Vanhuysse, S., Mboga, N., Wolff, E., Dujardin, S., Lennert, M., 2019. Modelling the Wealth Index of Demographic and Health Surveys within Cities Using Very High-Resolution Remotely Sensed Information. Remote Sensing, 11(21), 2543.

Geurs, K. T., Van Wee, B., 2004. Accessibility evaluation of land-use and transport strategies: review and research directions. Journal of Transport geography 12(2), 127-140.

Houet, T., Hubert-Moy, L., 2006. Modeling and projecting land-use and land-cover changes with Cellular Automaton in considering landscape trajectories: An improvement for simulation of plausible future states. EARSeL eProceedings, European Association of Remote Sensing Laboratories 5(1), 63-76.

Huff, D. 1964. Defining and Estimating a Trading Area. Journal of Marketing 28(3), 34-38.
Inglada, J., Vincent, A., Arias, M., Tardy, B., Morin, D., Rodes, I., 2017. Operational high resolution land cover map production at the country scale using satellite image time series. Remote Sensing, 9(1), 95

Jean, N., Burke, M., Xie, M., Davis, W. M, Lobell, D. B., Ermon, S., 2016. Combining satellite imagery and machine learning to predict poverty. Science, 353(6301),790-794.

Li, X., Yeh A.G.O., 2002. Neural-network-based cellular automata for simulating multiple land use changes using GIS, International Journal of Geographical Information Science 16(4), 323-343

Mossoux, S., Kervyn, M., Soulé, H., Canters, F., 2018. Mapping Population Distribution from High Resolution Remotely Sensed Imagery in a Data Poor Setting. Remote Sensing 10(9), 1409

Reilly, W. J., 1931. The law of retail gravitation. New York, Knickerbocker Press.

Stewart, J. Q., 1942. Measure of the Influence of a Population at a Distance, Sociometry 5(1), 63-71.

Wegener, M., 2004. Overview of land-use transport models. Handbook of transport geography and spatial systems 5, 127 146

Wegener, M., Fuerst, F., 2004. Land-Use Transport Interaction: State of the Art. SSRN Electronic Journal.

Wilson, A. G., 1967, A statistical theory of spatial distribution models, Transportation Research 1(3), 253-269

Wilson, A. G., 1998. Land-use/transport interaction models: Past and future. Journal of transport economics and policy, 326.

Zhang, L., Zhang, L., Du, B., 2016. Deep learning for remote sensing data: A technical tutorial on the state of the art. IEEE Geoscience and Remote Sensing Magazine 4(2), 22-40. 